ticularly in $S$ agalactiae infections. In the early postpartum period, fever in the mothers was significantly less likely in the patients offered vaginal disinfection, a reduction from $7 \%$ in those douched using saline compared with $3 \%$ in those disinfected using chlorhexidine. A lower occurrence of urinary tract infections also was observed: $6 \%$ in the saline group as compared with $3 \%$ in the chlorhexidine group $(P<.01)$.

This prospective controlled trial demonstrated that vaginal douching with $0.2 \%$ chlorhexidine during labor can significantly reduce both maternal and early neonatal infectious morbidity. The squeeze bottle procedure was simple, quick, and well-tolerated.

FROM: Stray-Pedersen B, Bergan T, Hafstad A, Normann E, Grogaard J, Vangdal M. Vaginal disinfection with chlorhexidine during childbirth. Int J Antimicrob Agents 1999;12:245-251.

\section{Effectiveness of Live, Attenuated Intranasal Influenza Virus Vaccine}

A recent study by Nichol and colleagues concluded that, among healthy adults, a live, attenuated influenza vaccine delivered intranasally not only helps prevent serious illness but also saves money.

In a randomized, double-blinded, placebo-controlled trial of 4,561 healthy adults aged 18 to 64 , investigators found that recipients of intranasally administered trivalent, live, attenuated influenza virus (LAIV) vaccine were as likely to experience one or more febrile illnesses as placebo recipients during peak outbreak periods ( $13.2 \%$ for vaccine vs $14.6 \%$ for placebo). However, vaccination significantly reduced the numbers of severe febrile illnesses $(18.8 \%$ reduction) and febrile upper respiratory tract illnesses (23.6\% reduction). Vaccination also led to fewer days of illness across all illness syndromes $(22.9 \%$ reduction for febrile illnesses; $27.3 \%$ reduction for severe febrile illnesses), fewer days of work lost (17.9\% reduction for severe febrile illnesses; $28.4 \%$ reduction for febrile upper respiratory tract illnesses), and fewer days with healthcareprovider visits $(24.8 \%$ reduction for severe febrile illnesses; $40.9 \%$ reduction for febrile upper respiratory tract illnesses). Use of prescription antibiotics and over-the-counter medications was also reduced across all illness syndromes. Vaccine recipients were more likely to experience runny nose or sore throat during the first 7 days after vaccination, but serious adverse events between the groups were not significantly different.

The match between the type $\mathrm{A}(\mathrm{H} 3 \mathrm{~N} 2)$ vaccine strain and the predominant circulating virus strain (A/Sydney/05/97[H3N2]) for the 1997/98 season was poor, suggesting that LAIV provided substantial crossprotection against this variant influenza $A$ virus strain. The authors concluded that intranasal trivalent LAIV vaccine was safe and effective in healthy, working adults in a year in which a drifted influenza $A$ virus predominated.

FROM: Nichol KL, Mendelman PM, Mallon KP, Jackson LA, Gorse GJ, Belshe RB, et al. Effectiveness of live, attenuated intranasal influenza virus vaccine in healthy, working adults: a randomized controlled trial. JAMA 1999;282:137-144.

\section{Gastrointestinal Endoscopic Reprocessing Practices in the United States}

Patient infection from contaminated gastrointestinal (GI) endoscopes generally can be attributed to failure to follow appropriate reprocessing guidelines. Recently, the Food and Drug Administration recommended a 45-minute exposure of GI endoscopes to $2.4 \%$ glutaraldehyde solutions heated to $25^{\circ} \mathrm{C}$. Simultaneously, the American Society for Gastrointestinal Endoscopy (ASGE), the American Gastroenterological Association, and the Society of Gastroenterology Nurses and Associates endorsed a reprocessing guideline that emphasized manual precleaning and recommended a 20 -minute exposure to a $2.4 \%$ glutaraldehyde solution at room temperature. Since then, little information has become available regarding actual reprocessing practices in the United States.

Cheung and colleagues mailed a questionnaire regarding endoscopic disinfection practices to 730 randomly selected members of the ASGE; 294 (40\%) responded. Appropriate manual cleaning (suctioning detergent through the accessory channel and brushing the channel and valves) was reported by $91 \%$ of respondents; $70 \%$ then used automated reprocessors for disinfection or sterilization. Glutaraldehyde was the most widely used chemical disinfectant; $85 \%$ used glutaraldehyde as one of their primary disinfectants. The most commonly used disinfection time with $2.4 \%$ glutaraldehyde was 20 minutes $(83.9 \%)$ followed by 45 minutes (11.4\%). Only $24 \%$ of users of $2.4 \%$ glutaraldehyde heated their solution; $60 \%$ of centers tested disinfectant concentration daily or more frequently; $74 \%$ sterilized nondisposable forceps before use; $29 \%$ of centers reused disposable endoscopic accessories (which are more frequently disinfected rather than sterilized). Twelve respondents reported cases of endoscopic cross-infection.

The authors note that a significant minority of endoscopy centers still do not completely conform to recent ASGE, American Gastroenterological Association, and the Society of Gastroenterology Nurses and Associates guidelines on disinfection, and they may not be appropriately disinfecting GI endoscopes. Rigid adherence to recommended guidelines is strongly encouraged to ensure patient safety.

FROM: Cheung RJ, Ortiz D, DiMarino AJ Jr. GI endoscopic reprocessing practices in the United States. Gastrointest Endosc 1999;50:362-368. 


\section{The School of Medicine at the University of Washington}

is recruiting a full-time faculty member at the Assistant or Associate Professor level to lead the program in Infection Control at the University of Washington Medical Center. The closing date for applications is December 10, 1999.

Although the School and Medical Center are seeking a person at the Assistant or Associate Professor rank, under unusual circumstances and commensurate with the qualifications of the individual, appointment may be made at the rank of Professor. Desirable credentials include national board certification in the candidate's specialty and ABIM eligibility in infectious diseases or advanced training in epidemiology, microbiology or related fields. Must have a strong clinical background with training in hospital epidemiology with emphasis on infection control. The selected individual will direct infection control activities of both ambulatory and inpatient care at the University of Washington Medical Center (including aspects of the new Seattle Cancer Care Alliance) and will participate in the clinical and teaching activities of their academic department. Development or continuation of an infection control or hospital epidemiology research program is desirable.

\section{Send C.V. to:}

Henry Rosen, M.D., Associate Chair

Department of Medicine, Box 356420

University of Washington, Seattle, WA 98195-6420

The University of Washington is building a culturally diverse faculty and strongly encourages applications from female and minority candidates. The University is an Equal Opportunity/Affirmative Action employer.

\section{Expand Your Horizons in Epidemiology}

\author{
Take An Inside Look At Cedars
}

For resources and challenges that will keep you on the leading edge of your field, take an inside look at Cedars-Sinai Medical Center. We're a world-class tertiary, acute care teaching medical center offering supportive management, a variety of continuing education opportunities and the assistance of a career counselor.

\section{NURSE EPIDEMIOLOGIST}

Join our team and provide expert leadership in infection control and epidemiology. You will develop and implement didactic programs as well as provide direction, consultation and development in relation to infection control and epidemiology. In addition, you will plan, coordinate and implement a system for prevention, surveillance, investigation and control of infection. Requires a Bachelor's degree (Master's preferred), CA RN license and CIC. Must have 3-5 years acute hospital experience, (preferably including one year as a nurse epidemiologist). Strong verbal/written English, basic computer and database management skills are essential

We offer a competitive salary and benefits package. Please fax or mail your resume to: Cedars-Sinai Medical Center, Attn: Harry KaKliKian (include Job \#1981956), 8723 Alden Dr., HR Dept., Room 110, Los Angeles, CA 90048, FAX: (310) 423-0374. AA/EEO.

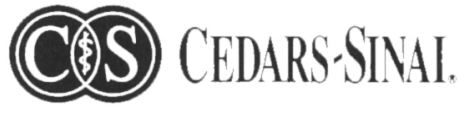



\begin{tabular}{|c|c|c|}
\hline 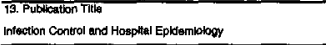 &  & \\
\hline 15. Extent and Nemure of Checulation & 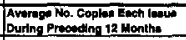 & 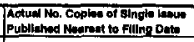 \\
\hline a. Towi Number of Coplose (Nat Pmot Run) & Q.487 & 8,633 \\
\hline  & 0 & a \\
\hline 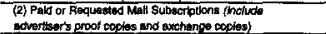 & 6,312 & 5,962 \\
\hline  & 5.912 & 5.962 \\
\hline 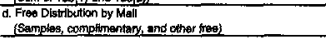 & 439 & 442 \\
\hline 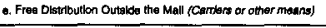 & 272 & 352 \\
\hline  & 705 & 794 \\
\hline 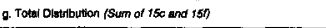 & 8.017 & 0.158 \\
\hline  & 470 & 477 \\
\hline (2) Foturns from News Agents & o & 0 \\
\hline 1. Total (Sum of 15g. 1Sh(t), and XSh(2)) & 6.487 & 6,2039 \\
\hline  & Ba\% & $B \%$ \\
\hline \multicolumn{3}{|c|}{  } \\
\hline \multicolumn{2}{|c|}{ 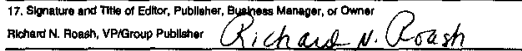 } & Date \\
\hline \multicolumn{3}{|c|}{  } \\
\hline
\end{tabular}

